International Journal of Child, Youth and Family Studies (2014) 5(1): 144-157

\title{
SUICIDE PREVENTION IN CHILD WELFARE: THE QUEBEC EXPERIENCE PROTECTING CHILDREN AND STAFF
}

\author{
Kees Maas
}

\begin{abstract}
In the year 2000, the Child Welfare System of Quebec implemented a Provincial Suicide Prevention Protocol. The goal of this Protocol was two-pronged: (a) heightened client safety, security, and treatment; and (b) heightened support to front line staff and better protection against vicarious traumatisation. From the vantage point of a participant-observer, the author uses Batshaw Youth and Family Centres as an example to illustrate the implementation and evolution of this Protocol. More specifically, the aim of this article is to demonstrate the benefits that clients and staff gain from such a Protocol. Further attention will be given to the impact this Protocol has on front line staff and second level team members intervening with suicidal clients, considerations that lessen the risks of vicarious traumatisation, as well as professional burnout. Statistics show there has been a decrease in suicidal gestures and attempts over the past decade following the Protocol's implementation. Finally, the supports and debriefing foreseen in the Protocol are appreciated by staff, and further supports such as clinical consultation, (peer) supervision, and continuing education have been implemented.
\end{abstract}

Keywords: suicide prevention, mental health screening, child welfare, vicarious traumatisation

Author's note: There are no conflicts of interest or sources of funding to be reported.

Kees Maas, Ph.D. is clinical psychologist with Batshaw Youth and Family Centres, 4039 Tupper Street, Westmount, Province of Quebec, Canada, H3Z 1T5, E-mail: kees_maas@ssss.gouv.qc.c 
International Journal of Child, Youth and Family Studies (2014) 5(1): 144-157

\section{Context and Clinical Profile of the Clientele}

Child Welfare in the Province of Quebec, Canada, is organized by 17 administrative regions, under the umbrella of "Centre jeunesse” (youth centre). The Centres jeunesse fall under the Quebec Ministry of Health and Social Services. These centres were created in the early 1990s and brought together different social services available to children and adolescents, including Centres for Social Services and Youth Rehabilitation Centres. Thus, all child and adolescent social services (adoption, child protective services, residential and foster placement) were regrouped under one roof.

The clientele of the Centres jeunesse shows a wide diversity of social and mental health problems. First and foremost the families struggle with problems related to parenting, substance abuse, mental health, unemployment, housing, and often poverty. Children come to the attention of the child protection services for reasons of neglect (the primary reason they are signalled to youth protective services), different types of abuse, family violence, abandonment, and for the older children (10 and older), conduct disorders. The Centres jeunesse service a highly vulnerable clientele presenting a wide variety of mental health problems, including non-suicidal self-injury (NSSI), suicidal ideation, suicidal attempts, and suicide.

In a given year at Batshaw Youth and Family Centres, an average of 42 situations require high levels of supervision due to severe suicidal behaviour and approximately three times as many situations require the involvement of mental health specialists.

\section{The Inception of the Protocol}

Following a coroner's inquiry of suicides among youth serviced by the Centres jeunesse, a provincial Suicide Prevention Protocol (Protocol d'intervention en situation de problématique suicidaire dans les Centres jeunesse) was created through a collective effort (Association des Centres Jeunesse, 2000). This effort brought together the Association of Centres jeunesse of Quebec (ACJQ), the professional licensing board of Physicians (College des Médecins du Québec), the provincial association of hospitals (Association des Hôpitaux du Québec), and the provincial association of local centres for community health and social services (Association des CLSC et des CHSLD du Québec).

The Protocol defined three interdependent levels of intervention. The first level refers to first line interveners of the Centres jeunesse (educators, social workers, and managers) who have the mandate to screen for suicidal risk and, in close collaboration with the second and third levels, to put in place safety and intervention plans. The second level specifically created by this Protocol consists of general practitioners, nurses, and psychologists, whose roles are to assess suicidal risk, decide on specific levels of supervision required, assist the first line interveners in establishing safety and intervention plans, support the first line through debriefing of incidents (suicidal attempts) and postvention following suicides. The second level interveners also refer to the third 
International Journal of Child, Youth and Family Studies (2014) 5(1): 144-157

level of intervention, comprised of child psychiatrists, whose mandate is to assess suicidal risk, provide psychiatric diagnoses and intervention recommendations.

This Protocol brought about a clarification of the mandates of the different interveners involved and established clearer communication pathways and referral processes in cases of suicidal risk. It further led to the creation of the second level team in the organigram of the Centre jeunesse. This required the establishment of service contracts with general practitioners, redefining mandate assignments for nurses and psychologists already in the service of the Centre jeunesse, and in many regions the need to establish resource sharing with the Local Community Services Centres (CLSC). Attracting general practitioners to the Centre jeunesse was difficult in many cases because of the lack of general practitioners in the Province and the pressure of the global health system on reduced resources.

The official language of Quebec is French, so the initial documents referred to here are in French. The author has been involved as a consultant-trainer in the initial dissemination of the Protocol to all Centres jeunesse of the Province, but mostly in its planning, implementation and follow-up at Batshaw Youth and Family Centres. This Centre jeunesse is the only one to provide services in English to families, children, and youth from the Island of Montreal, as well as to English speaking clients requiring services that other regions of the Province cannot provide in English.

\section{Implementation of the Provincial Suicide Prevention Protocol at Batshaw Youth and Family Centres (BYFC)}

\section{Challenges and Adjustments}

Given the involvement of the author with a provincial task force on suicide prevention in the early and mid-1990s, the BYFC had already started to create policies and procedures, identify appropriate screening tools, develop interview questions to be used, and establish a better working relationship with child psychiatry. When the Provincial Protocol came out, a review and modification of these documents and necessary adjustments to the practice were required. The first document produced after the introduction of the provincial protocol was the BYFC policy (Batshaw Youth and Family Centres, 2001) with the following objectives:

1. Detect clients at risk of suicidal behaviour, determine the level of urgency and severity of suicidal behaviour, and act accordingly.

2. Prevent the occurrence of suicide or any forms of suicidal behaviour as well as decrease the risk of reoccurrence and possible contagion effect.

3. Increase the sensitivity and awareness of caregivers, families, and partners to the issue of depression and suicide and enlist their involvement in helping clients at risk.

4. Ensure that interveners and foster parents give systematic, immediate, and specific attention to all suicidal behaviours and that they are not left isolated in dealing with a situation of suicidal behaviour. 
International Journal of Child, Youth and Family Studies (2014) 5(1): 144-157

5. Provide a necessary level of supervision when a client at risk of suicidal behaviour is in placement (residential, foster care), pay particular attention to this risk in service and intervention plans, and foresee follow-up services, adequate support, and referral to appropriate resources.

6. Clarify all stages of interventions indicating the roles and responsibilities of BYFC personnel and foster parents with respect to the phenomenon of suicide.

7. Record, gather, and share relevant information concerning situations of suicidal behaviour.

8. Standardize BYFC's approach in order to provide continuity of clinical interventions in dealing with suicidal behaviour.

9. Re-establish stability amongst staff and clients in the event of a suicide attempt or completed suicide.

10. Manage the impact of a completed suicide within BYFC and with our partners.

The guiding principle of this policy is to ensure that suicidal risk is screened for, and handled appropriately, either through internal resources or external specialized resources. This aspect of the Protocol focuses on the safety, security, and treatment of clients.

The other important principle is to assure that no intervener remains isolated in dealing with such difficult situations, as well as to provide supports to family and others closely related to the suicidal person. In addition, these measures were taken in order to prevent contagion, particularly in cases of suicide. The vulnerability of suicide survivors has been well documented in the literature (Séguin, Kiely, \& Lesage, 1994; Séguin, Brunet, \& LeBlanc, 2006) and requires due attention, especially given a clientele already representing a host of risk factors associated with suicidal behaviour. This aspect of the BYFC policy refers to the Protocol's aim to acknowledge the inherent stress that suicidal behaviour brings to the staff and the need for the organization to provide supports in order to diminish the risks of vicarious traumatisation or professional burnout.

Secondly, parallel to the elaboration of the above policy, procedures were written in order to standardize the practice within Batshaw Youth and Family Centres regarding suicidal behaviour. One of the main functions of the procedures is to provide interveners with clear guidelines as to what to do when different types of suicidal behaviour occur. The procedures foresee the roles of different interveners in the organization, the communication channels, the recording of information in the case of suicidal ideation, suicidal ideation with a plan, a suicide attempt, and a suicide.

One of the initial challenges was to provide a support system to the front line staff during regular hours as well as outside regular hours (evenings, nights, and weekends). In order to cover 24 hours a day, and seven days a week, one idea was to have the small second level team take turns and be on call, above their habitual duties. This was deemed too great a burden on these professionals, and didn't necessarily make sense clinically. Any crisis occurring in the evening, night, or weekend would require first and foremost measures to assure the safety and security of the client. It so happened that the residential services of BYFC were creating a pool of duty officers, called on-site managers, to cover the evenings, nights, and weekends and thus were well placed to assist the front line staff in making decisions about heightened supervision or the necessity for risk assessment by the hospital system. All further assessment and setting up of intervention plans is better done during regular hours when both first and second level interveners 
International Journal of Child, Youth and Family Studies (2014) 5(1): 144-157

are on duty and can convene in order to collaborate on a common understanding of the case and of the treatment modalities.

The second level interveners provide support to the on-site managers through debriefing, ongoing consultation, and communication related to levels of supervision and crisis management strategies. Occasionally, the question of second line intervener availability outside of the regular hours resurfaces, particularly on the heels of demanding and emotionally intense situations often related to clients displaying chronic suicidal or self-harming behaviours. In these times, additional supports that are provided in a timely fashion can partly respond to the need expressed.

\section{Levels of Supervision}

One of the aims of the Suicide Prevention Protocol was to develop a common language to use when referring to suicidal ideation, a suicidal plan, and a suicide attempt, as well as to introduce clear levels of supervision accordingly. Every Centre jeunesse, and often residential programs and hospital programs, have their own terminology, such as "suicide watch" or "strict sight supervision" to indicate what structure and supervision to provide to a suicidal client. The Protocol introduced two levels of supervision required in the case of suicidal risk that contrast with the regular supervision generally applied to all youth in a given program. This regular supervision is referred to as the first level of supervision.

Level Two (Close supervision) requires visual contact at least every half-hour. When the youth concerned is in a location that is difficult for the supervising adult to access (e.g., washroom, shower), the frequency of the controls and the intensity of the supervision must increase. This may be accomplished by asking the youth to respond at shorter intervals to questions such as: "Is everything okay?" or "Are you okay in there?”. All necessary and possible changes can be made to the environment to make it safer: For example, personal belongings and furniture that pose a risk can be removed, and required alterations to the architectural layout, such as to doors and windows, can be undertaken to make the premises safer.

Level Three (Constant supervision) involves visual and auditory contact with the youth at all times. This involves "one-on-one" monitoring. As is the case for close supervision, this level requires that all necessary and possible changes be made to the environment to make it safer. Since it can only be exercised in locations where these overall conditions can be met, "security-type units" may sometimes be the only place where constant supervision can take place. This decision is made by the program manager or on-site manager.

\section{Collaboration with Child Psychiatry}

One other aspect of implementing the Protocol involved collaboration with Child Psychiatry. One of the irritants between the hospital network and the child welfare network had been the use of the Emergency Room (ER) as a way to get assessments of suicidal risk and general psychological functioning. The clarification of expectations regarding what an assessment in the ER can provide (e.g., assessment of suicidal risk) helped to create other 
International Journal of Child, Youth and Family Studies (2014) 5(1): 144-157

avenues for the more global assessment of psychological functioning, (e.g., determining a psychiatric diagnosis). Consequently, a service corridor was developed specifically for clients of Batshaw Youth and Family Centres. It used the paediatrician affiliated to our second level team for the referral, and the nurses who gathered all the information in the file, including information from the Batshaw psychologists and other professionals involved. This helped the child welfare interveners obtain more helpful and timely guidance in the organisation of their interventions. It was decided that the ER would only be used in the case of a need for immediate risk assessment.

\section{Agency Consultation and Approval}

The initial drafts of the policy and the procedures were submitted for consultation to the different departments and standing committees (multidisciplinary council, multicultural or multiracial committee) and were ultimately approved by the Batshaw Management committee (Batshaw Youth and Family Centres, 2001). The Protocol was introduced to the Division of Residential Services for Adolescents at first, because this division receives the Agency's most vulnerable clientele that is most prone to acting out. Over time, procedures for the other divisions were introduced in a staggered way.

\section{Constitution of Second Level Team}

The first step was to constitute the second level team and begin training. Initially, designated nurses and a psychologist were identified and updated about the policy and the procedures. The main focus was to clarify their tasks and involvement in the different stages of intervention in cases of suicidal behaviour, but also to provide them with a theoretical training regarding suicidal behaviour as symptoms associated to mood disorders, conduct disorders often in comorbidity with substance abuse disorders. The framework for this training was developed at a provincial level and then adapted, translated, and delivered by the author for the Agency.

\section{Training of Front Line Staff}

In a fairly short time period, all first level staff (educators and managers, as well as social workers affiliated exclusively to residential services) received one day of training, comprised of a theoretical component similar to the second level training, as well as an introduction to the policy. Participants were invited to participate in a personal exercise concerning their feelings, attitudes, and values regarding suicidal behaviour, followed by a discussion related to ethical and religious considerations, and the stance taken by the Agency that suicide in children and young people needs to be prevented with all our might. Thus, as professionals of our Agency, they are expected to adopt this value and stance.

Furthermore, in this training, the procedures are presented with a clear outline of role expectations and checklists of actions to undertake, depending on the type of suicidal behaviour presented by the client. The training proceeds further through experiential learning with vignettes

of situations where participants are required to assess the situation and identify the actions required 
International Journal of Child, Youth and Family Studies (2014) 5(1): 144-157

according to the outlined procedures. These initial trainings became the base for future trainings that were later developed to accompany the implementation of this protocol in the other divisions. Through the years, these trainings have become one of the requirements in the orientation of new staff. Over time, the handling of non-suicidal self-injuries became a separately identified part of the procedures and the trainings.

In the initial stages of this training, trainers encountered a lot of anxiety with the front line staff and a concern regarding the degree of training it takes to work with suicidal clients. The scope of the training was also to relay to the staff working directly with clients on a daily basis that many of the interventions required are of a psycho-educational nature and totally consistent with the base training and practice of educators. Furthermore, the focus was on clarifying the multidisciplinary collaboration and the support the second and third levels provide. With time, and the use of the procedures, the first line staff became more confident with their capacity to intervene as well as with the supports in place. Staff who participated in the training, after having already worked with suicidal clients and the new procedures, were more prepared, emotionally and professionally, to see these situations as an intricate part of their practice. Ongoing consultation with staff in the residential units provided the opportunity to refresh the theoretical components of this Protocol training. Consultation is also a forum to discuss specific interventions, clinical procedures, the usefulness of specifically designed individual crisis management plans, and not in the least occasions to discuss the emotional strain suicidal clients bring to the individual worker and to the team.

\section{Management of Supervision Levels}

The procedures of this Protocol foresee the possibility of heightening the level of supervision of a youth in care because of suicidal risk. A Level Two supervision is generally put in place when there is suicidal ideation, some thoughts about the possible means, but no plans. Any manager, including the on-site manager, can put in place the Level Two supervision. This level entails checking regularly (a minimum of every halfhour) with the youth about his or her state of mind, and more frequently if the youth is not visible during shower or washroom use. It doesn't entail, however, waking up youth while they are asleep.

One of the problems encountered with this level of supervision is the difficulty of carrying it over from one shift to the other, and the management of youth going into the community with this level (e.g., travelling to school). Especially if the level is maintained for several days, the practice has shown that it sometimes gets lost in the shuffle, particularly if the youth has gone out of obvious crisis mode and is settled in a quieter way. Several strategies have been used, certainly systematic shift exchange with a formal point on the agenda around levels of supervision. Helping educators with broaching the difficult subject of suicidal ideation has been another way to avoid oversight of the level. Level Two has been adapted to allow youth to maximize their attendance at activities that are meaningful and gratifying to them, such as school or team sports. It requires putting clear boundaries and expectations around the travel time and route, and regular checking in by the unit with the location where the youth is, for example by calling in when leaving and arriving. These arrangements always require a case-by-case consideration 
International Journal of Child, Youth and Family Studies (2014) 5(1): 144-157

and when discomfort about any of these activities persists, it is better to cancel when one is not sure the youth can handle either the travel or the activity itself in the light of his or her more vulnerable state.

The Protocol calls for a discussion with the second level team for a reduction of this level back to regular supervision and this decision is made conjointly between the first level team and second level team staff. There had been some confusion in the initial days of the Protocol around the role of the psychologist as opposed to the role of the nurse, in this process. The procedures clearly state that either one of these professionals can be involved in the lowering of this level.

Level Three can also be instituted by any manager, except that during regular hours it is done in consultation with the second level team. Level Three is used when there is a high risk of acting on the suicidal ideation, generally because the youth has a plan. Often the Level Three is used after a youth has gone into the hospital emergency because of a suicidal gesture or attempt. With time, the child psychiatrists became somewhat aware of the existence of these levels of supervision, but every situation necessitates discussion with the medical staff regarding the required need of supervision.

When a youth is placed on Level Three supervision, one staff is with the youth at all times. The staff keeps the youth engaged, which doesn't mean constant talk about suicide. However, the thoughts about suicide need regular examination, at least shift-by-shift. The youth is an essential source in this examination and is generally a good indicator of where he or she is at any given time. The educator uses his clinical judgment and the youth is also met by the second level staff, generally the nurse, more often present on the premises of the residential units. The changing of Level Three always requires a discussion between the second level professionals, as well as with the first level staff. Unlike Level Two, it is the psychologist who decides on a change of the level, after due consultation.

\section{Debriefing and Postvention}

In the course of the last decade, several situations of suicide attempts have required debriefing and in two cases postvention was required. Postvention is the specific process called for in cases of suicide. Two youth committed suicide months after their discharge upon reaching the age of 18. These last two youth had been under BYFC's care for an extended period of time, had been chronically suicidal, and were highly invested in by the youth care teams that worked with them. Other meetings resembling postvention meetings have occurred following violent or sudden deaths of current or former clients, and of staff.

In debriefing situations, generally two representatives of the second level team would intervene with the professionals as well as with the peers, and in several situations also with the family of the suicidal client or with families of other clients affected by the situation. The debriefing procedure is very close to Mitchell's critical incident stress debriefing developed in the 1970s (Mitchell, 1983). Small groups gather in order to allow fact sharing, thoughts and reactions, and to teach how the symptoms are in the realm of the normal in these circumstances. An important goal of the debriefing is to help people move on, get them back to a routine, identify 
International Journal of Child, Youth and Family Studies (2014) 5(1): 144-157

individuals who might require more support and possibly need referral to outside help through the employee assistance program or other resources.

For the second level interveners it was pivotal to have follow-up meeting(s) after any debriefing or postvention intervention. The second level interveners were all seasoned clinicians; however, not all of them had been exposed to this particular kind of clinical work and thus the initial debriefing or postvention sessions were learning experiences. The literature helped to prepare, but the subsequent debriefing of the debriefing sessions helped to integrate the theory and the practice for the second level interveners. One of the challenges with setting up debriefing sessions with residential intervention teams is the timeliness. Ideally, they are done within a framework of 24 to 72 hours. It is not always easy to get all the professionals together within this time frame. The constraints of work responsibilities, scheduling, part-time work, etc., didn't always permit the availability of two second level professionals and many debriefing sessions ended up being run by one professional. In addition, it was often required that these second level professionals extend their normal work schedules. Following the debriefing, supports were put in place to help the professional after such solo interventions and provide additional support in the follow-up.

The postvention sessions were not structurally different from the debriefing sessions, but the suicides affected far more people, both staff, youth, and their families. The sessions with the youth always happened before the staff sessions. As much as possible staff members were present in the sessions with the youth, mainly in order to be able to follow up later with the client group or with individual clients. Therefore, some staff members would have been going to the process first in the presence of some of their clients and then later in company of their colleagues.

\section{The Benefits and Weaknesses of the Protocol to the Safety, Security, and Treatment of Clients}

The main benefit of the Suicide Prevention Protocol has been the systematic screening for suicidal behaviour. Over the years, however, this protocol has expanded to include a systematic screening of global mental health. This screening has attracted attention to the presence of disorders as well as to the required treatments. The systematic use and application of the different levels of supervision, depending on the level of suicidal risk, has brought a greater sense of safety and security, both for children and their families, as well as staff.

These procedures give clear guidelines for intervention as well as for channels of communication. The Protocol has allowed for a tighter and clearer collaboration with the local child psychiatry network. The use of the Emergency Room has also diminished and now clients have scheduled assessments through our service corridor. These assessments go beyond a risk assessment, and provide more information and recommendations that can be worked into the intervention and service plans for clients.

The weaknesses of this Protocol are related to some of the benefits. First of all, the systematic screening underscores different types of treatment, some of which are relatively easy to put in place, while others cannot be delivered in a timely manner due to a lack of resources (e.g., psychotherapy). Secondly, following up on crises with regular consultation regarding issues of 
International Journal of Child, Youth and Family Studies (2014) 5(1): 144-157

mental health and ensuing intervention strategies to the treating team would be desirable, but cannot be systematically available for resource reasons, on the one hand, and on the other, once the crisis is over, the motivation to pursue these issues is lessened at the team level. Thirdly, the Level Two supervision has been problematic when extending over several shifts or several days, especially when the behaviour of the child or youth has subsided and is attracting less attention. Efforts have been made to include a discussion of the level of supervision in every shift change, but the most successful method has not yet been found to assure a more flawless continuity. Fourthly, clear communication about the different events and interventions through the involvement of both first and second level staff remains a challenge. The electronic file system in use would allow the centralization of everybody's progress notes, but departmental procedures are not always aligned and the streamlining of communication channels that requires major attention is currently in the works.

\section{Exposure to Suicidal Behaviour and its Impact on Staff}

The youth presenting with suicidal ideations and attempts requires more structure and supervision and thus the highly structured secure residential units are more likely to receive these youth. In addition, staff in these residential units are often far more exposed to the stress that is part and parcel of working with suicidal clients. Unfortunately, one has no control over the frequency of occurrence, nor on the number of suicidal clients one might have in a secure unit at any given time. Work in the field of child welfare exposes interveners to a variety of sources contributing to the suffering of their clients and to the different ways this suffering translates into often disturbing and self-destructive behaviour. Individual resources, professional training, ongoing education, and clinical supervision are means available to interveners to deal with the stress inherent to their daily work. Professionals working in higher structured secure units that receive clients prone to suicidal or self-harming behaviour are at greater risk for vicarious traumatisation (McCann \& Pearlman, 1990; Pearlman \& Saakvitne, 1995). Dunkley and Whelan (2006) discuss concepts related to vicarious traumatisation such as professional burnout, secondary traumatic stress disorder, and compassion fatigue. Vicarious traumatisation seems to best describe the specific impact that work with trauma victims can have on interveners.

Several of the residential teams of secure units have been heavily burdened by the presence of one or more chronic suicidal clients, and the disclosure of the life stories behind the suicidal behaviour brings its share of exposure to the trauma in these children's and youths' lives that can lead to feelings of being overwhelmed. Vicarious traumatisation refers to a transformation in the trauma worker's inner experience resulting from empathic engagement with the trauma material of a client (Figley, 1995, 1999; Lewis \& Bensmihen, 2009). In order to avoid vicarious traumatisation or avoid professional burnout, a mix of personal and organizational strategies is required (Rothchild, 2006; Trippany, White Kress, \& Wilcoxon, 2004). Each individual can seek the best work-rest-play balance for themselves, maintain a good social support network, both professionally and personally, take care of one's physical health, identify personal limits, and acknowledge the personal distress that work can bring. Spirituality can be another source of personal support. Organizational supports are in the realm of providing appropriate training as well 
International Journal of Child, Youth and Family Studies (2014) 5(1): 144-157

as access to continuing education; provide workers with manageable caseloads, consultation, and consistent supervision as well a respectful and supportive environment.

Despite the best knowledge of these different strategies, individuals do react differently. Because of the stress of working in the units described above, these reactions may include booking off shifts or even going on sick leave. This scenario can then create more pressure on the remaining staff. Moreover, the replacement staff members often have less work experience and can be more vulnerable to vicarious traumatisation. At the organizational level, additional supports can be offered by the second level team. Over the past decade, experience has shown that this kind of help was often not asked for in a timely manner, and was only requested after exposure to a series of stressful incidents. The recuperation after exceptionally stressful shifts, with the occurrence of suicide attempts or recurrent serious self-injury, takes more than a single debriefing. The best remedy constitutes a series of "normal” shifts with their normal stress.

Helping a youth through one suicidal crisis is one thing; but dealing with a chronic suicidal youth is very different and much more demanding. The constant threat of a possible tragic gesture drains a lot of energy. How can a team best be accompanied in these stressful times? It is known that stress rises in an exponential fashion when a unit receives a second or a third suicidal client in the same way as described by Rutter (1987), given the impact of the accumulation of stressors on the capacity to develop resilience. Of course, the resilience of a team depends on its composition, but also on the presence of leadership, as well as the pressure it has confronted in its recent past. The staffing of residential unit teams follows the rules of collective agreements and solutions such as the rotation of personnel or temporary reassignment cannot be implemented easily. Other solutions such as temporary or permanent mandate changes of the units or redirection of clients are equally difficult or impossible to implement given the limited resources available. Despite being well trained and capable of understanding the dynamics of such clients, there is a limit to the staff's tolerance to this stress. Some individuals are more resistant, depending on their personal resources and the circumstances in their lives. Personal circumstances can bring temporary reduction of the tolerance threshold in the workplace. Some of these variables are beyond institutional control, but the organization has to maximize its strategies to provide supports and aim at the reduction of the stress related to suicidal clients. For instance, a more timely presence of heightened clinical support can be one of these strategies.

Professionals that provide this type of support expose themselves to the risk of getting overburdened as well. Hence, the importance of the optimal distance from the practice that allows the provision of support to the front line staff without getting sucked into the same maelstrom.

\section{The Benefits and Weaknesses of the Protocol for the Protection of Staff from Vicarious Victimisation}

The procedures provide guidelines for intervention and channels of communication among the different levels of intervention and the main goal is to avoid the isolation of interveners. The supports foreseen by the procedures, such as the implication of the nurses and psychologists, are very helpful, but the front line workers can still feel isolated during evenings, nights and 
International Journal of Child, Youth and Family Studies (2014) 5(1): 144-157

weekends, when these professionals are neither on the premises nor available by phone. The rotation of on-call duties often leads to situations where front line workers have to repeat background information and recent events to different professionals, leading to frustration and also loss of useful information. Ongoing follow-up around a specific client by a designated second level professional has been a way to provide ongoing support to residential teams. Each highly structured secure unit has a specific professional attached as consultant. This professional can provide ongoing training on issues of mental health, suicidal behaviour, non-suicidal self-injury, as well as participation in clinical discussions aimed at improving intervention strategies.

\section{Conclusion}

Suicide prevention in Child Welfare cannot be dissociated from careful screening for mental health concerns. The vulnerable population of the Centres jeunesse has many of the risk factors associated with the occurrence of self-harm and of suicidal behaviour. Early detection and the treatment of the underlying mental disorders as well as promotion of global mental health through educational programming are all ways to prevent suicidal behaviour. Clinical support to the front line staff and professional assessment of the suicidal risk and the mental disorders by mental health professionals is another condition to prevent suicide. Furthermore, adequate training to front line staff in terms of theoretical understanding as well as professional intervention strategies adds to the confidence staff have in their capacity to help these vulnerable youth. Last but not least, ongoing supervision, clinical consultation, and peer support and supervision will help front line staff to maintain their professional and emotional capacity to face the daily stressors and the high demands that come with work with these vulnerable children and youth. 
International Journal of Child, Youth and Family Studies (2014) 5(1): 144-157

\section{References}

Association des Centres Jeunesse. (2000). Protocole d'intervention en situation de problématique suicidaire dans les Centres jeunesse [Intervention protocol regarding suicidal behaviour in youth centres]. Montréal: Association des Centres Jeunesse.

Batshaw Youth and Family Centres (BYFC). (2001, February). The handling of suicidal behaviour. Policy of suicide prevention at Batshaw Youth and Family Centres, Division of Professional Services. Montréal: Author.

Dunkley, J., \& Whelan, T. (2006). Vicarious traumatization: Current status and future directions. British Journal of Guidance and Counselling, 34(1), 107-116. http://dx.doi.org/10.1080/03069880500483166

Figley, C. R. (1995). Compassion fatigue as secondary traumatic stress disorder: An overview. In C. R. Figley (Ed.), Coping with secondary traumatic stress disorders in those who treat the traumatized (pp. 1-20). New York: Brunner/Mazel Publishers.

Figley, C. R. (1999). Compassion fatigue: Toward a new understanding of the cost of caring. In B. H. Stamm (Ed.), Compassion fatigue: Secondary traumatic stress: Self-care issues for clinicians, researchers and educators (2nd ed., pp. 3-28). Lutherville, MD: Sidran Press.

Lewis, W., \& Bensmihen, K. (2009, November). “No, no, don’t worry about me, I’m fine. Meanwhile...”: Dealing with vicarious trauma in child welfare workers. Batshaw Youth and Family Centres. Professional Day, 2009. Montréal: Batshaw Youth and Family Centres.

McCann, I. L., \& Pearlman, L. A. (1990). Vicarious traumatization: A framework for understanding the psychological effects of working with victims. Journal of Traumatic Stress, 3(1), 131-149. http://dx.doi.org/10.1002/jts.2490030110

Mitchell, J. T. (1983). When disaster strikes.... The critical incident stress debriefing process. Journal of Emergency Medical Services, 13(11), 49-52.

Pearlman, L. A., \& Saakvitne, K. W. (1995). Trauma and the therapist: Countertransference and vicarious traumatization in psychotherapy with incest survivors. New York: W.W. Norton.

Rothchild, B. (2006). Help for the helper. The psychophysiology of compassion fatigue and vicarious trauma. New York: Norton.

Rutter, M. (1987). Psychosocial resilience and protective mechanisms. American Journal of Orthopsychiatry, 57(3), 316-331.http://dx.doi.org/10.1111/j.1939-0025.1987.tb03541.x 
International Journal of Child, Youth and Family Studies (2014) 5(1): 144-157

Séguin, M., Kiely, M. C., \& Lesage, A. (1994). After suicide: A unique mourning experience? Santé Mentale au Québec, 19(2), 63-82.

Séguin, M., Brunet, A., \& LeBlanc, L. (2006). Intervention en situation de crise et en contexte traumatique [Intervention in situations of crises and related to trauma]. Montreal: Les éditions de la Chenelière.

Trippany, R. L., White Kress, V. E., \& Wilcoxon, S. A. (2004). Preventing vicarious trauma: What counselors should know when working with trauma survivors. Journal of Counseling \& Development, 82(1), 31-37.http://d.doi.org/10.1002/j.1556-6678.2004.tb00283.x 